This paper has been accepted for the 29th Mediterranean Conference on Control and Automation in Bari, June 22-25 2021.

Please cite the paper as: L. Ballotta, M. R. Jovanović, and L. Schenato,

"Optimal Network Topology of Multi-Agent Systems subject to Computation and Communication Latency", 29th Mediterranean Conference on Control and Automation (MED), 2021.

\title{
Optimal Network Topology of Multi-Agent Systems subject to Computation and Communication Latency
}

\section{(with Proofs)}

\author{
Luca Ballotta $^{1}$, Mihailo R. Jovanović ${ }^{2}$ and Luca Schenato ${ }^{1}$
}

\begin{abstract}
We study minimum-variance feedback-control design for a networked control system with retarded dynamics, where inter-agent communication is subject to latency. We prove that such a design can be solved efficiently for circular formations and compute near-optimal control gains in closed form. We show that the centralized control architecture is in general suboptimal when the communication delays increase with the number of links, and propose a control-driven optimization of the network topology.
\end{abstract}

\section{Index Terms}

Communication latency, Decentralized control, Feedback latency, Minimum-variance control, Multi-agent systems, Networked control systems.

\section{INTRODUCTION}

Large-scale networked control systems have been scaling up both in terms of number of agents and spatial distribution over the latest years, taking advantages of new communication protocols for massive systems, e.g., 5G [5], [17], and of advances in embedded electronics [25] enhancing the computing performance of the network agents [23], [26]. Indeed, distributed sensing and computation represent the true asset of such systems, whose tasks would be otherwise infeasible. Since when the concept of multi-agent systems was introduced to the literature, decentralized control techniques have been developed to adapt classical approaches to network applications [3].

This work has been partially funded by the CARIPARO Foundation Visiting Programme "HiPeR" and by the Italian Ministry of Education, University and Research (MIUR) through the PRIN project no. 2017NS9FEY entitled "Realtime Control of 5G Wireless Networks: Taming the Complexity of Future Transmission and Computation Challenges" and through the initiative "Departments of Excellence" (Law 232/2016). The views and opinions expressed in this work are those of the authors and do not necessarily reflect those of the funding institutions.

${ }^{1}$ L. Ballotta and L. Schenato are with the Department of Information Engineering, University of Padova, 35131 Padova, Italy \{ballotta, schenato\}@dei.unipd.it

${ }^{2}$ M. R. Jovanović is with the Ming Hsieh Department of Electrical and Computer Engineering, University of Southern California, Los Angeles, CA 90089 USA mihailo@usc.edu 
A few examples can be found in control of vehicular formations [7], [10] and of traffic congestion in vehicular networks [12], ground and aerial robot swarms [16], [22], [27] and UAV-UGV cooperative teams [1].

One of the main issues related to multi-agent systems is latency, which may be caused by inter-agent communication, limited computational power, or delayed sensing and actuation. In particular, limited bandwidth and the so-called finite channel capacity pose a serious limitation to the scalability of large-scale systems and when the information exchange among agents involves bulky data, as with multimedia or in federated learning [20], [24].

Several works in the literature deal with decentralized control in the presence of latency. [8], [9] design control laws with convergence conditions for platoons with various network topologies. [28] studies consensus for multi-agent systems with delayed dynamics and noisy sensors. [21] is concerned about finite-time stability of discrete-time systems with neutral delay. Control-theoretic literature is also fairly rich in delay systems. For example, [4] finds sufficient conditions for uniform stability of linear delay differential equations.

Drawing inspiration from such line of work, we design minimum-variance control of networked systems subject to communication latency; in particular, we focus on the trade-off arising when the delays depend on the amount of links among the agents. Such situation may occur, for example, when the bandwidth available for inter-agent communication is fixed a priori [11], so that additional links lead to a delay increase for all data transmissions, or when multi-hop communication causes data to accumulate non-negligible delays [14]. In particular, we shall see that centralized control gets outperformed if the latency increases too quickly with the network topology, overtaking the benefit given by a larger amount of feedback information. To the best of our knowledge, this approach is new to the existing literature.

To approach such control design with delays, we consider circular formations. Such systems are deployed for, e.g., source seeking by smart mobile sensors [6], [19] or target tracking by robots [2], [18]. In particular, we focus on consensus of scalar states, which may represent the agents' 2D orientation or a sensed quantity present in the environment.

\section{I-A Paper Outline and Main Results}

The paper is organized as follows. Section II introduces the networked-system model and preliminary results on circular formations and delay systems. Section III formalizes the control problem: namely, we optimize the feedback gains in order to minimize the scalar variance of 
the system at steady state. The solution is presented in Section III-A for the single-parameter formulation (all gains equal) and in Section III-B for the multiple-parameter formulation. In particular, we prove that a near-optimal solution with multiple parameters coincides with a singleparameter configuration. In Section IV] we discuss the performance of the network topology under varying communication latency, proposing a control-driven optimization for such topology that takes into account the rate of delay variation. Specifically, we show that using all communication links (centralized control) is in general suboptimal. Finally, concluding remarks are drawn in Section V, together with future work directions.

\section{SETUP}

\section{II-A System Model}

We consider a circular formation composed of $N$ agents, where each agent $i \in[N]$ is modeled as a single integrator:

$$
d \bar{x}_{i}(t)=\bar{u}_{i}(t) d t+d \bar{w}_{i}(t)
$$

where $\bar{x}_{i}(t) \in \mathbb{R}$ is the state of agent $i$ at time $t$, the control input $\bar{u}_{i}(t) \in \mathbb{R}$ affects the state variation at time $t$, and $\bar{w}_{i}(t)$ is standard Brownian noise. Each agent computes its control input $\bar{u}_{i}(t)$ exploiting its own (measured) state and possibly others received via wireless in a symmetric fashion.

Assumption 1. Agent $i$ receives measurements from the agent pairs $\ell$ positions ahead and behind, $\ell=1, \ldots, n<N / 2$. All inter-agent transmissions are subject to the communication delay $\tau_{n}=f(n) \tau_{\min }$, where the rate $f(n)$ is a non-decreasing sequence and $\tau_{\text {min }} \in \mathbb{R}_{+}$is a constant.

Remark 1. Even though the linear rate $f(n)=n$ looks more natural, other rates are possible, e.g., if the measurements are processed or compressed upon acquisition. In general, $f(n)$ may be estimated or learned from transmission data.

The control input has the following structure:

$$
\begin{gathered}
\bar{u}_{i}(t)=u_{d}(t)+u_{i}(t) \\
u_{i}(t)=-\sum_{\ell=1}^{n}\left(k_{\ell} y_{i, i-\ell}\left(t-\tau_{n}\right)+k_{\ell} y_{i, i+\ell}\left(t-\tau_{n}\right)\right)
\end{gathered}
$$


where $u_{d}(t)$ is the desired reference (feedforward control input) at time $t, u_{i}(t)$ is the feedback control input for agent $i, k_{\ell}$ is $\ell$-th the feedback gain, $\ell=1, \ldots, n$, and

$$
y_{i, i \pm \ell}(t)= \begin{cases}\bar{x}_{i}(t)-\bar{x}_{i \pm \ell}(t), & 0<i \pm \ell \leq N \\ \bar{x}_{i}(t)-\bar{x}_{i \pm \ell \mp N}(t), & \text { otherwise }\end{cases}
$$

are the state differences computed with the received measurements. All agents use the same feedback gains $\left\{k_{1}, \ldots, k_{n}\right\}$.

System (1)-(2) can be modeled in vector-matrix form as

$$
d \bar{x}(t)=\mathbb{1}_{N} u_{d}(t) d t-K \bar{x}\left(t-\tau_{n}\right) d t+d \bar{w}(t)
$$

where $\mathbb{1}_{N}$ is the vector of all ones of dimension $N$ and $d \bar{w}(t) \sim \mathcal{N}\left(0, I_{N} d t\right)$. In particular, the feedback

matrix $K \in \mathbb{R}^{N \times N}$ is circulant and can be written as

$$
\begin{gathered}
K=K_{f}(k)+K_{f}^{\top}(k) \\
K_{f}(k)=\operatorname{circ}\left(\sum_{\ell=1}^{n} k_{\ell},-k_{1}, \ldots,-k_{n}, 0, \ldots, 0\right)
\end{gathered}
$$

where $\operatorname{circ}\left(a_{1}, \ldots, a_{N}\right)$ denotes the circulant matrix with vector $a=\left[a_{1}, \ldots, a_{N}\right]$ as first row. For example, if $N=6$ and $n=2$,

$$
K=\left[\begin{array}{llllll}
* & * & * & 0 & * & * \\
* & * & * & * & 0 & * \\
* & * & * & * & * & 0 \\
0 & * & * & * & * & * \\
* & 0 & * & * & * & * \\
* & * & 0 & * & * & *
\end{array}\right]
$$

Let us decompose the state as $\bar{x}(t)=\bar{x}_{m}(t)+x(t)$, where $\bar{x}_{m}(t)$ has all components equal to the mean of $\bar{x}(t)$ and $x(t)$ represents the mismatches of each agent's state:

$$
\begin{array}{cc}
\bar{x}_{m}(t)=P \bar{x}(t), & x(t)=\Omega \bar{x}(t) \\
P \doteq \frac{\mathbb{1}_{N} \mathbb{1}_{N}^{\top}}{N}, & \Omega \doteq I-P
\end{array}
$$


We study the dynamics of such two orthogonal components independently. The mean-vector dynamics equation reads

$$
d \bar{x}_{m}(t)=P d \bar{x}(t)=\mathbb{1} u_{d}(t) d t+P d \bar{w}(t)
$$

because the term with coordinate differences given by (2b) lives in the kernel of $P$. The noise in (9) can be compensated by adding a further feedback term. The error dynamics is

$$
\begin{aligned}
d x(t)=\Omega d \bar{x}(t) & =-\Omega K \bar{x}\left(t-\tau_{n}\right) d t+\Omega d \bar{w}(t)= \\
& =-K \Omega \bar{x}\left(t-\tau_{n}\right) d t+d w(t)= \\
& =-K x\left(t-\tau_{n}\right) d t+d w(t)
\end{aligned}
$$

where $w(t)=\Omega \bar{w}(t)$. From now on, we focus on system $(10)$.

\section{II-B Eigenvalue Analysis}

We exploit symmetry to diagonalize $K=T \Lambda T^{\top}$, with $T$ orthogonal and $i$-th eigenvalue $\lambda_{i}=\Lambda_{i i}$ equal to [13]

$$
\lambda_{i}=2 \sum_{\ell=1}^{n} k_{\ell}\left(1-\cos \left(\frac{2 \pi(i-1) \ell}{N}\right)\right), i=1, \ldots, N
$$

With the change of basis $\tilde{x}(t)=T^{\top} x(t)$, the dynamics reads

$$
d \tilde{x}_{i}(t)=-\lambda_{i} \tilde{x}_{i}\left(t-\tau_{n}\right) d t+d \tilde{w}_{i}(t)
$$

where the noise differential $d \tilde{w}(t)$ has covariance matrix

$$
T^{\top} \Omega T=(\Omega T)^{\top}(\Omega T)=\left[\begin{array}{c|c}
0 & 0 \\
\hline 0 & I_{N-1}
\end{array}\right]
$$

because the leftmost eigenvector in $T$, associated with $\lambda_{1}=0$, belongs to the kernel of $\Omega$, and all the other eigenvectors in $T$ are also in $\Omega$. In particular, the coordinate $\tilde{x}_{1}(t)$ (i.e., the mean of $x(t)$ ), has trivial dynamics and does not affect system (10), whose state coordinates converge to random variables with expectation equal to $\tilde{x}_{1}(0)$. For the sake of simplicity, in the following we assume that $x(0)$ is zero mean. 


\section{II-C Minimum-Variance Control of Scalar Delay Systems}

Let us consider the stochastic retarded differential equation

$$
\begin{aligned}
d x(t) & =-a x(t-\tau) d t+d w(t), \quad t \geq 0 \\
x(t) & =x_{0}(t), \quad t \in[-\tau, 0]
\end{aligned}
$$

where $x(t), a \in \mathbb{R}, \tau \in \mathbb{R}_{+}$is the delay, $w(t)$ is standard Brownian noise, and $x_{0}(t), t \in[-\tau, 0]$, is the initial condition.

Theorem 1. [15] Eq. (14) admits a steady-state solution $x_{s s}(t)$ if and only if

$$
a \in\left(0, \frac{\pi}{2 \tau}\right)
$$

If it exists, $x_{s s}(t)$ is the unique and it is a zero-mean Gaussian process with variance

$$
\sigma_{s s}^{2}=\int_{0}^{\infty} x_{d}^{2}(s) d s=\frac{1+\sin (a \tau)}{2 a \cos (a \tau)}
$$

where $x_{d}(t)$ is the so-called fundamental solution of the deterministic equation corresponding to (14):

$$
\begin{aligned}
& \dot{x}(t)=-a x(t-\tau), \quad t \geq 0 \\
& x(t)=\delta(t), \quad t \in[-\tau, 0]
\end{aligned}
$$

If (15) holds, $x_{d}(t)$ and its derivative are exponentially stable.

Let us consider now the system with dynamics

$$
d x(t)=u(t) d t+d w(t)
$$

and control input $u(t)=-\lambda x(t-\tau)$. We want to choose the gain $\lambda$ in order to minimize the steady-state variance of $x(t)$. If $\tau=0$, the (infeasible) optimum is $+\infty$ and $\lambda$ can be increased arbitrarily. Instead, if $\tau>0$, the following holds.

Lemma 1. The variance $\sigma_{s s}^{2}$ given by (16) with constraint (15) is strictly convex in a.

Proof. See Appendix A.

Proposition 1. Let system (18) have input $u(t)=-\lambda x(t-\tau)$ with delay $\tau>0$ and steady-state 


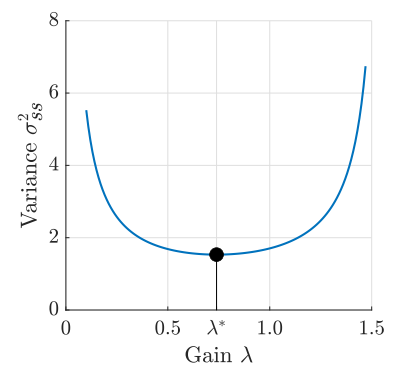

Fig. 1: Variance $\sigma_{s s}^{2}$ as a function of the gain $\lambda(\tau=1)$.

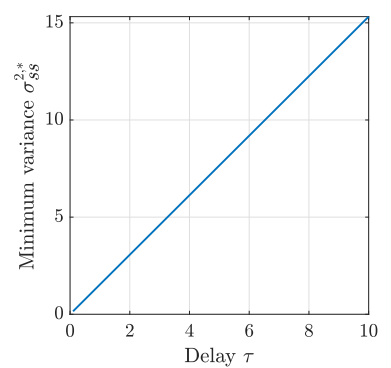

Fig. 2: Minimum variance $\sigma_{s s}^{2, *}$ as a function of $\tau$.

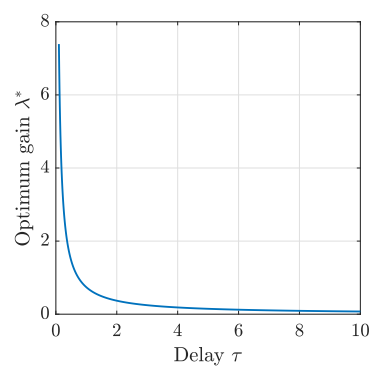

Fig. 3: Optimum gain $\lambda^{*}$ as a function of the delay $\tau$.

variance of $x(t)$

$$
\sigma_{s s}^{2}(\lambda)=\frac{1+\sin (\lambda \tau)}{2 \lambda \cos (\lambda \tau)}
$$

Then, the minimum-variance control problem

$$
\underset{\lambda \in\left(0, \frac{\pi}{2 \tau}\right)}{\arg \min } \sigma_{s s}^{2}(\lambda)
$$

has the following unique solution:

$$
\lambda^{*}=\frac{\beta^{*}}{\tau}, \quad \sigma_{s s}^{2, *} \doteq \sigma_{s s}^{2}\left(\lambda^{*}\right)=\frac{1+\sin \beta^{*}}{2 \cos ^{2} \beta^{*}} \tau
$$

with $\beta^{*}=\cos \beta^{*}$.

Proof. See Appendix B.

Fig. 1 3 show the variance (19) and the optimal variance $\sigma_{s s}^{2, *}$ and gain $\lambda^{*}$ as functions of the delay, respectively.

\section{Optimization of Feedback Gains}

In this section, we aim to optimize the feedback gains in order to minimize the steady-state scalar variance of $x(t)$.

Problem 1. Given system (10) with communication as per Assumption 1, find the feedback gains $k^{\top}=\left[k_{1}, \ldots, k_{n}\right]$ minimizing the steady-state scalar variance of $x(t)$ :

$$
\underset{k \in \mathbb{R}^{n}}{\arg \min } \sigma_{s s}^{2}(x)
$$

where $\sigma_{s s}^{2}(x) \doteq \mathbb{E}\left[\left\|x_{\infty}\right\|^{2}\right]$ and $x_{\infty} \doteq \lim _{t \rightarrow \infty} x(t)$. 


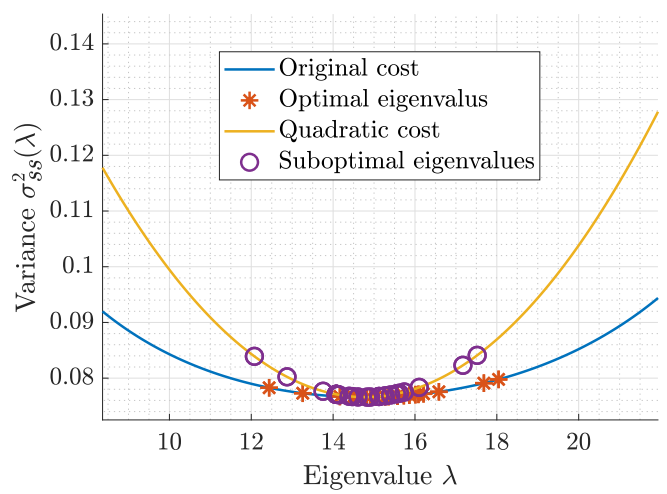

Fig. 4: Cost functions in (24) and in (25) about the minimum.

Problem reformulation. In virtue of the change of basis presented in Section II-B, we write

$$
\mathbb{E}\left[\left\|x_{\infty}\right\|^{2}\right]=\mathbb{E}\left[x_{\infty}^{\top} x_{\infty}\right]=\mathbb{E}\left[\tilde{x}_{\infty}^{\top} T^{\top} T \tilde{x}_{\infty}\right]=\mathbb{E}\left[\left\|\tilde{x}_{\infty}\right\|^{2}\right]=\sum_{i=2}^{N} \sigma_{s s}^{2}\left(\lambda_{i}\right)
$$

where the contribution of $\lambda_{1}=0$ is neglected in virtue of the trivial dynamics of $\tilde{x}_{1}(t)$. Problem (22) can be rewritten as

$$
\begin{array}{ll}
\underset{k \in \mathbb{R}^{n}}{\arg \min } & \sum_{i=2}^{N} \sigma_{s s}^{2}\left(\lambda_{i}(k)\right) \\
\text { s.t. } & \lambda_{M}(k)<\frac{\pi}{2 \tau_{n}}
\end{array}
$$

where $\lambda_{M}=\max _{i} \lambda_{i}$ and the constraint ensures that the steady-state solution exists (c.f. (15)). Notice that, in virtue of (11) and Lemma 1, problem (24) is convex and the optimal gains can be found numerically. To also get analytical intuition, we shift to the (sub-optimal) quadratic optimization of the variance arguments, i.e., the eigenvalues of $K$ :

$$
\begin{array}{ll}
\underset{k \in \mathbb{R}^{k}}{\arg \min } & \sum_{i=2}^{N}\left(\lambda_{i}(k)-\lambda^{*}\right)^{2} \\
\text { s.t. } & \lambda_{M}(k)<\frac{\pi}{2 \tau_{n}}
\end{array}
$$

The intuition behind the above reformulation is that the quadratic cost function in (25) approximates well the variance in (24) about the minimum (up to scaling and translation). In particular, the spectrum of $K$ needs to be "close" to the optimal gain $\lambda^{*}$ because the variance grows quickly with the smallest and largest eigenvalues (c.f. Fig. 1). Fig. 4 compares the two cost functions in (24)-28) for $N=50, n=10$ and $\tau_{n}=0.1$, together with their optimal eigenvalues. 
Remark 2. Problem 1 may be regularized to penalize excessive control efforts. For example, any norm of the vector $k$ can be added to the costs in 24-25) without affecting convexity. This also allows to consider a delay-free dynamics in (10).

\section{III-A Single Parameter}

We first impose that all feedback gains are equal to the parameter $\alpha>0$, such that

$$
\begin{gathered}
K=K_{f}(\alpha)+K_{f}^{\top}(\alpha) \\
K_{f}(\alpha)=\operatorname{circ}\left(\left[\begin{array}{lllllll}
n \alpha & -\alpha & \ldots & -\alpha & 0 & \ldots & 0
\end{array}\right]\right)
\end{gathered}
$$

The original variance minimization (22) boils down to

$$
\begin{array}{ll}
\underset{\alpha \in \mathbb{R}_{+}}{\arg \min } & \sum_{i=2}^{N} \sigma_{s s}^{2}\left(\lambda_{i}(\alpha)\right) \\
\text { s.t. } & \lambda_{M}(\alpha)<\frac{\pi}{2 \tau_{n}}
\end{array}
$$

and the quadratic approximation (25) becomes

$$
\begin{array}{ll}
\underset{\alpha \in \mathbb{R}_{+}}{\arg \min } & \sum_{i=2}^{N}\left(\lambda_{i}(\alpha)-\lambda^{*}\right)^{2} \\
\text { s.t. } & \lambda_{M}(\alpha)<\frac{\pi}{2 \tau_{n}}
\end{array}
$$

Theorem 2. The solution of 28 is $\tilde{\alpha}^{*}=\frac{\lambda^{*}}{2 n+1}$.

Proof. See Appendix C.

Given (21), Theorem 2 implies that the near-optimal gain (control effort) decreases as $1 / n \cdot 1 / f(n)$, where the first factor is related to the denser communication topology (more feedback information) and the second, which is ruled by the delay increase at rate $f(n)$, is a direct consequence of the constraint (15) that embeds a stability condition.

\section{III-B Multiple Parameters}

We now let $k_{j} \neq k_{i}$ such that $k$ is any vector in $\mathbb{R}^{n}$.

Theorem 3. The solution of (25) is $\tilde{k}^{*}=\mathbb{1}_{n} \tilde{\alpha}^{*}$. 


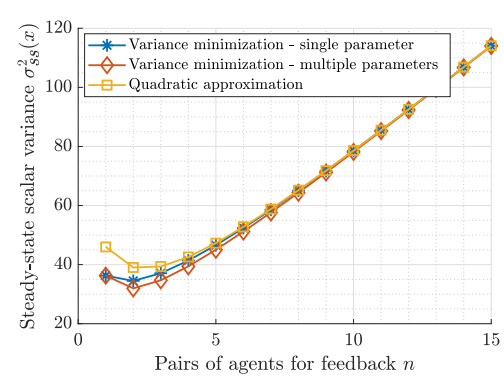

(a) Delay rate $f(n)=n$.

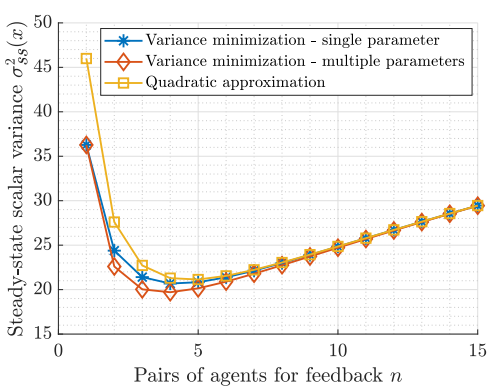

(b) Delay rate $f(n)=\sqrt{n}$.

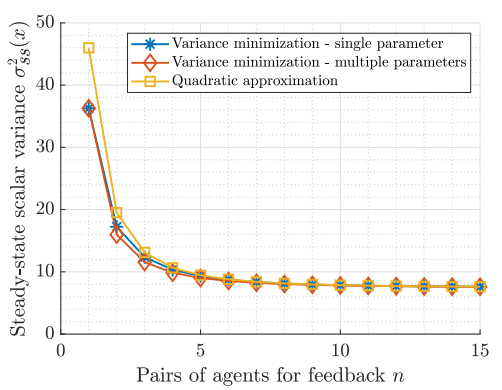

(c) Delay rate $f(n) \equiv 1$.

Fig. 5: Variance obtained from Problem 1 with single (blue) and multiple parameters (red) and approximation (28) (yellow).

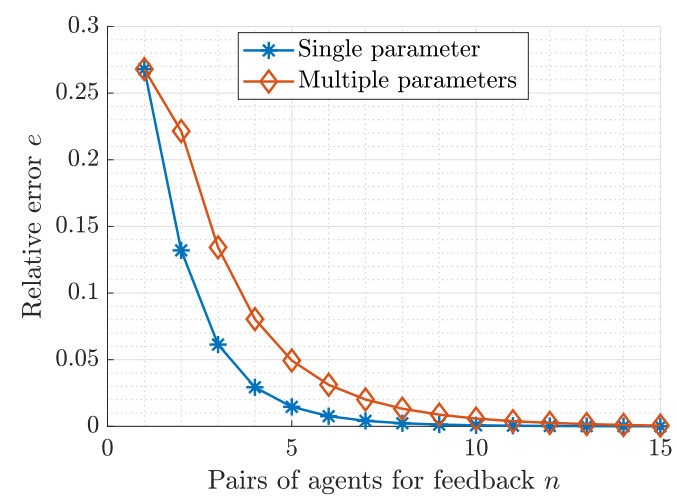

Fig. 6: Relative error due to the quadratic approximation (28).

Proof. See Appendix D.

Theorem 3 states that optimizations (25) and (28) coincide, i.e., choosing the same gain for all agents is near-optimal even if multiple gains are allowed. Fig. 5 compares the scalar variances obtained from single-parameter (27) and multiple-parameter (22) variance minimization and from the quadratic approximation 28 with $N=50, \tau_{\min }=0.01$ and several rates $f(n)$. Fig. 6 shows the relative error (variance increase) due to the quadratic approximation (28), defined as

$$
e \doteq \frac{\tilde{\sigma}_{x, s s}^{2, *}-\sigma_{x, s s}^{2, *}}{\sigma_{x, s s}^{2, *}}
$$

where $\sigma_{x, s s}^{2, *}$ is the optimal value of (27) or (22) and $\tilde{\sigma}_{x, s s}^{2, *}$ is the scalar variance obtained with the near-optimal gain. 


\section{Discussion: The Role of LATEnCy in DeCEntralized Control}

The gains obtained by solving Problem 1 or its quadratic approximation (28) depend on the system parameters, such as the pairs of communicating agents $n$ and the delay $\tau_{n}$. It follows that the (near-)optimal variance also depends on such parameters. The following result quantifies such dependence for the approximated problem (28).

Proposition 2. The scalar variance $\tilde{\sigma}_{x, s s}^{2, *}$ can be written as

$$
\tilde{\sigma}_{x, s s}^{2, *}(n)=C^{*}(n) f(n) \tau_{\min }
$$

where $C^{*}(n)$ does not depend on $f(n)$ and can be computed exactly. Also, the optimization of the communication topology

$$
n^{*}=\underset{n \in \mathbb{N}}{\arg \min } \tilde{\sigma}_{x, s s}^{2, *}(n)
$$

has solution $n^{*} \leq n_{\max } \doteq\lfloor N / 2\rfloor$.

Proof. See Appendix E

Numerical computations show that $C^{*}(n)$ is submodular decreasing: such result is hard to prove, because $C^{*}(n)$ is the sum of non-monotone addends. As a consequence, $30-31$ imply that, when the communication delay increases with the amount of feedback measurements, decentralized control can outperform centralized: in general, the communication topology is optimized when the number of links is smaller than the maximum available. Also, the optimum $n^{*}$ depends on the rate $f(n)$ in a "non-increasing" fashion: namely, slower rates yield larger optima (e.g., $f(n)=\sqrt{n}$ yields an optimum which is larger than with $f(n)=n$, see Fig. 5a 5 b. In particular, using all available agents for feedback (centralized control) is optimal if the delay is constant, see Fig. 5c 1

As shown in the proof of Proposition 2, the key to formula 30 is that the near-optimal gain $\tilde{\alpha}^{*}$ is linear in $\lambda^{*}$ through a coefficient $c^{*}(n)$ that only depends on $n$. Even though proving the same relationship also for the optimal gain $\alpha^{*}$ analytically is hard, numerical evidence suggests that this is indeed the case. For example, Fig. 7 shows the ratio $\alpha^{*} / \lambda^{*}$, which is not affected by the specific value of $\tau_{\min }$ and hence is consistent with the guess $\alpha^{*}=c^{*}(n) \lambda^{*}$. Also, simulations

\footnotetext{
${ }^{1}$ Although the same holds true with $\tau_{n} \equiv 0$, in this case the computations in Appendix E cannot be applied.
} 


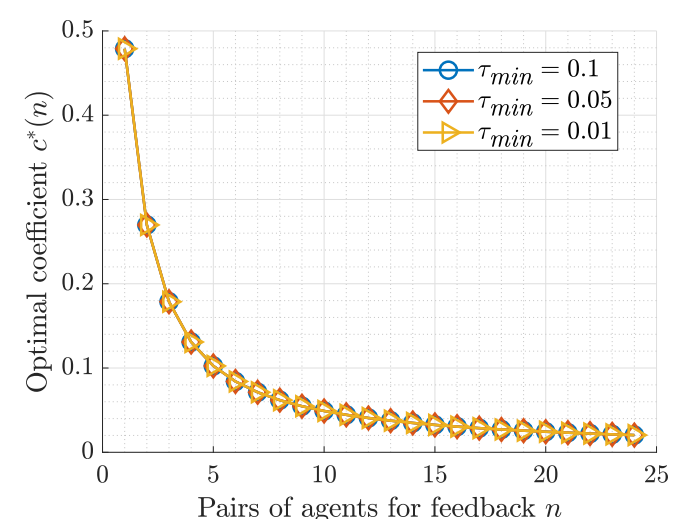

Fig. 7: Ratio $\alpha^{*} / \lambda^{*}$ with different values of $\tau_{\min }$.

show that the relative gap depicted in Fig. 6 is independent of the rate $f(n)$, reinforcing such hypothesis: indeed, if $\sigma_{x, s s}^{2, *}=K^{*}(n) f(n) \tau_{\min }$, 29] can be rewritten as

$$
e=\frac{C^{*}(n)}{K^{*}(n)}-1
$$

This suggests that the minimum variance obtained from 22 27 can also be optimized over $n$. The optimal network topology needs to trade the feedback information (maximized by the centralized architecture) for the latency (minimized by fully distributed control). In particular, $n^{*}$ represents a threshold beyond which the benefit of adding links is overpowered by the delay increase in the retarded dynamics.

Remark 3. All above results assume a fixed system size $N$ for ease of notation. In general, as one can notice in the proof, the coefficient $C^{*}(n)$ is parametric in $N$, and therefore the optimal variance $\tilde{\sigma}_{x, s s}^{2, *}$ and $n^{*}$ in (31) also depend on $N$.

Remark 4. Eq. (30) shows that $n^{*}$ does not depend on $\tau_{\min }$. In particular, the optimal communication topology is only determined by how the delay grows, i.e., the rate $f(n)$.

\section{CONCLUSIONS}

In this paper, motivated by decentralized control for circular formations, we design minimumvariance control gains for a noisy dynamical system subject to feedback latency with a strong dependence on the communication topology, i.e., the number of agents involved in the feedback loops. Also, near-optimal gains are found, which are characterized by a simple closed-form expression and whose performance almost attains the optimum as the communication topology 
becomes denser. When the feedback latency grows with the number of communication links, e.g., if the available bandwidth for all agents is fixed a priori, we show that centralized control is in general suboptimal, and that the optimal decentralized architecture can be found explicitly. Future improvements to this work might involve general graph topology, a more complex model for the agent dynamics (e.g., double integrator), or heterogeneous agents with, possibly, different computational capabilities and delays.

\section{APPENDIX A \\ PROOF OF LEMMA 1}

Because $\tau$ does not impact convexity, we assume $\tau=1$. The second derivative of $\sigma_{s s}^{2}(a)$, written in (33), is positive if

$$
\begin{aligned}
a^{3} \cos ^{3} a+2 a \cos ^{3} a+2 a^{3} \cos a \sin a & +2 a \cos ^{3} a \sin a+ \\
+ & 2 a^{3} \cos a \sin ^{2} a-2 a^{2} \cos ^{2} a \sin a-2 a^{2} \cos ^{2} a>0
\end{aligned}
$$

The constraint (15) ensures that $a, \cos a$ and $\sin a$ are positive. We now consider three cases for $a$ and show that the negative terms in (34) are always outbalanced by the positive terms.

$a>1:$

$$
\begin{gathered}
2 a^{3} \cos a \sin ^{2} a>2 a^{2} \cos ^{2} a \sin a \\
2 a^{3} \cos a \sin a>2 a^{2} \cos ^{2} a
\end{gathered}
$$

$\cos a<a \leq 1$ :

$$
\begin{gathered}
2 a^{3} \cos a \sin a>2 a^{2} \cos ^{2} a \sin a \\
2 a^{3} \cos a \sin ^{2} a+2 a \cos ^{3} a \geq 2 a^{3} \cos a>2 a^{2} \cos ^{2} a
\end{gathered}
$$

$\frac{d^{2} \sigma_{s s}^{2}}{d a^{2}}=\frac{(1+2 \sin a+a \cos a-\cos (2 a)) a^{2} \cos ^{2} a+(a-\cos a+a \sin a-\sin a \cos a)\left(2 a^{2} \sin a-2 a \cos a\right) \cos a}{a^{4} \cos ^{4} a}$ 
$a \leq \cos a:$

$$
\begin{aligned}
2 a \cos ^{3} a \sin a & \geq 2 a^{2} \cos ^{2} a \sin a \\
2 a \cos a^{3} & \geq 2 a^{2} \cos ^{2} a
\end{aligned}
$$

\section{APPENDIX B}

\section{Proof of Proposition 1}

Uniqueness of $\lambda^{*}$ follows from strict convexity. Setting the derivative of the variance equal to zero, we have

$$
\frac{d \sigma_{s s}^{2}}{d \lambda}=\frac{\tau \lambda-\cos (\tau \lambda)+\tau \lambda \sin (\tau \lambda)-\cos (\tau \lambda) \sin (\tau \lambda)}{2 \lambda^{2} \cos ^{2}(\tau \lambda)}=0
$$

which is verified if and only if

$$
(1+\sin (\tau \lambda))(\tau \lambda-\cos (\tau \lambda))=0
$$

Because of the constraint in (20), (42) is satisfied if and only if $\tau \lambda=\cos (\tau \lambda)$. It is then sufficient to apply the change of variable $\beta=\tau \lambda$, which admits a unique solution in $(0, \pi / 2)$.

\section{APPENDix C \\ PROOF OF THEOREM 2}

According to (11), we first rewrite each eigenvalue as $\lambda_{i}=g_{i} \alpha$. The cost function in (28) can then be rewritten as

$$
C(\alpha)=\sum_{i=2}^{N}\left(g_{i} \alpha-\lambda^{*}\right)^{2}
$$

We first exploit strict convexity to find the global minimum of (43). To this aim, we set

$$
C^{\prime}(\alpha)=2 \sum_{i=2}^{N} g_{i}\left(g_{i} \alpha-\lambda^{*}\right)=0
$$

which admits the unique solution

$$
\tilde{\alpha}^{*}=\frac{\sum_{i=2}^{N} g_{i}}{\sum_{i=2}^{N} g_{i}^{2}} \lambda^{*}
$$


The coefficients $g_{i}$ are the eigenvalues of $K$ when $\alpha=1$. Because the eigenvalues of the circulant matrix $\operatorname{circ}(a)$ are the Discrete Fourier Transform (DFT) of $a$, we can write

$$
\begin{gathered}
\sum_{i=2}^{N} g_{i}=\sum_{i=1}^{N} g_{i}=N \sum_{i=1}^{N} r_{i}=2 N n \\
\sum_{i=2}^{N} g_{i}^{2}=\sum_{i=1}^{N} g_{i}^{2}=N \sum_{i=1}^{N} r_{i}^{2}=N\left(4 n^{2}+2 n\right)
\end{gathered}
$$

where $r$ is the first row of $K$, (46) comes from the definition of inverse DFT and (47) from Plancherel theorem. The final expression of $\tilde{\alpha}^{*}$ follows by substituting (46)-47) in (45).

We now need to check if such solution satisfies the constraint. We first note that $\lambda^{*}<\pi / 4 \tau_{n}$ by studying the sign of the derivative in (41) at $\lambda=\pi / 4 \tau_{n}$, in virtue of convexity. We then have the following relations for the maximum eigenvalue $\tilde{\lambda}_{M}$ :

$$
\begin{aligned}
\tilde{\lambda}_{M}^{*} & =g_{M} \tilde{\alpha}^{*}=2 \tilde{\alpha}^{*}\left(n-\sum_{j=1}^{n} \cos \left(\frac{2 \pi M j}{N}\right)\right)= \\
& =2 \frac{\lambda^{*}}{2 n+1}\left(n-\sum_{j=1}^{n} \cos \left(\frac{2 \pi M j}{N}\right)\right)<2 \frac{\pi}{4 \tau_{n}} \frac{2 n}{2 n+1}<\frac{\pi}{2 \tau_{n}}
\end{aligned}
$$

\section{APPENDIX D}

\section{PROOF OF THEOREM 3}

Exploiting linearity of the DFT and Plancherel theorem, problem (25) can be recast as follows:

$$
\begin{aligned}
& \tilde{k}^{*}=\underset{k \in \mathbb{R}^{n}}{\arg \min }\left\|r(k)-\lambda^{*} e_{1}\right\|_{2}^{2} \\
& \text { s.t. } \quad \lambda_{i} \in \mathcal{S}\left(\tau_{n}\right), i=2, \ldots, N
\end{aligned}
$$

where $r^{\top}(k)$ is the first row of $K$ and $e_{\ell}$ is the $\ell$-th canonical vector in $\mathbb{R}^{N}$. Consider now the problem

$$
\begin{array}{ll}
x^{*}= & \underset{x \in \mathbb{R}^{N}}{\arg \min }\left\|x-e_{\ell}\right\|_{2}^{2} \\
\text { s.t. } & x_{i}=0 \quad \forall i \in \mathcal{I} \subset[N] \\
& \sum_{i \notin \mathcal{I}} x_{i}=0
\end{array}
$$

with $\ell \in[N] \backslash \mathcal{I}$ fixed and $|\mathcal{I}|=N-n-1$ with $n>0$. We show that all nonzero coordinates (i.e., not in $\mathcal{I}$ ) of $x^{*}$ coincide (possibly except $x_{\ell}^{*}$ ), which implies that the optimal gains $k_{i}^{*}$ in 
$r\left(k^{*}\right)$ are equal. Assume there exists $j \notin \mathcal{I} \cup\{\ell\}$ such that $x_{j} \neq x_{i}=\bar{x}$ for all $i \notin \mathcal{I} \cup\{j, \ell\}$. The cost of $x$ is

$$
C_{x}=\left(x_{\ell}-1\right)^{2}+(n-1) \bar{x}^{2}+x_{j}^{2}
$$

Let $\tilde{x}$ such that $\tilde{x}_{\ell}=x_{\ell}, \tilde{x}_{i}=\overline{\tilde{x}}$ for all $i \notin \mathcal{I} \cup\{\ell\}$. We have

$$
\begin{aligned}
& x_{\ell}=-x_{j}-\sum_{i \neq \ell, j} x_{i}=-x_{j}-(n-1) \bar{x} \\
& \tilde{x}_{\ell}=-\sum_{i \neq \ell} \tilde{x}_{i}=-n \overline{\tilde{x}}
\end{aligned}
$$

and the cost associated with $\tilde{x}$ is

$$
C_{\tilde{x}}=\left(\tilde{x}_{\ell}-1\right)^{2}+n \overline{\tilde{x}}^{2}=\left(x_{\ell}-1\right)^{2}+\frac{\left[(n-1) \bar{x}+x_{j}\right]^{2}}{n}
$$

We then have the following chain of inequalities:

$$
\begin{aligned}
{\left[(n-1) \bar{x}+x_{j}\right]^{2} } & <n\left[(n-1) \bar{x}^{2}+x_{j}^{2}\right] \\
2(n-1) \bar{x} x_{j} & <(n-1)\left(\bar{x}^{2}+x_{j}^{2}\right) \\
0 & <\left(\bar{x}-x_{j}\right)^{2}
\end{aligned}
$$

which implies $C_{\tilde{x}}<C_{x}$.

The statement follows from optimality of $\tilde{\alpha}^{*}$ in $(28)$.

\section{APPENDIX E \\ PROOF OF PROPOSITION 2}

The proof follows straightforward manipulations of the analytical expression of $\tilde{\sigma}_{x, s s}^{2, *}$. From (23), we have

$$
\tilde{\sigma}_{x, s s}^{2, *}=\sum_{i=2}^{N} \sigma_{s s}^{2}\left(\tilde{\lambda}_{i}^{*}\right)
$$

where $\tilde{\lambda}_{i}^{*}=g_{i}(n) \tilde{\alpha}^{*}$ are the near-optimal eigenvalues (c.f. Appendix C). Consider the formula in Theorem 2 for the near-optimal gain $\tilde{\alpha}^{*}$, which we rewrite as $\tilde{\alpha}^{*}=c^{*}(n) \lambda^{*}$. Then, each 
variance $\sigma_{s s}^{2}\left(\tilde{\lambda}_{i}^{*}\right)$ can be written as follows:

$$
\begin{aligned}
\sigma_{s s}^{2}\left(\tilde{\lambda}_{i}^{*}\right) & =\frac{1+\sin \left(\tilde{\lambda}_{i}^{*} \tau_{n}\right)}{2 \tilde{\lambda}_{i}^{*} \cos \left(\tilde{\lambda}_{i}^{*} \tau_{n}\right)}= \\
& =\frac{1+\sin \left(g_{i}(n) c^{*}(n) \lambda^{*} \tau_{n}\right)}{2 g_{i}(n) c^{*}(n) \lambda^{*} \cos \left(g_{i}(n) c^{*}(n) \lambda^{*} \tau_{n}\right)}= \\
& =\frac{1+\sin \left(a_{i}^{*}(n) \beta^{*}\right)}{2 a_{i}^{*}(n) \beta^{*} \cos \left(a_{i}^{*}(n) \beta^{*}\right)} \tau_{n}=C_{i}^{*}(n) \tau_{n}
\end{aligned}
$$

where (21) has been used, $a_{i}^{*}(n) \doteq g_{i}(n) c^{*}(n)$ and $C_{i}^{*}(n)$ multiplies $\tau_{n}$ in the third line of (56). The scalar variance can then be written as

$$
\tilde{\sigma}_{x, s s}^{2, *}=\sum_{i=2}^{N} C_{i}^{*}(n) \tau_{n}=C^{*}(n) f(n) \tau_{\text {min }}
$$

where $C^{*}(n) \doteq \sum_{i=2}^{N} C_{i}^{*}(n)$.

\section{REFERENCES}

[1] Barbara Arbanas, Antun Ivanovic, Marko Car, Matko Orsag, Tamara Petrovic, and Stjepan Bogdan. Decentralized planning and control for uav-ugv cooperative teams. Autonomous Robots, 42(8):1601-1618, 2018.

[2] Lara Brinón Arranz, Alexandre Seuret, and Carlos Canudas De Wit. Translation control of a fleet circular formation of auvs under finite communication range. In Proceedings of the 48h IEEE Conference on Decision and Control (CDC) held jointly with 2009 28th Chinese Control Conference, pages 8345-8350. IEEE, 2009.

[3] Lubomír Bakule. Decentralized control: An overview. Annual Reviews in Control, 32(1):87 - 98, 2008.

[4] Leonid Berezansky, Josef Diblík, Zdeněk Svoboda, and Zdeněk Šmarda. Simple uniform exponential stability conditions for a system of linear delay differential equations. Applied Mathematics and Computation, 250:605 - 614, 2015.

[5] Andrea Biral, Marco Centenaro, Andrea Zanella, Lorenzo Vangelista, and Michele Zorzi. The challenges of m2m massive access in wireless cellular networks. Digital Communications and Networks, 1(1):1-19, 2015.

[6] Lara Briñón-Arranz, Luca Schenato, and Alexandre Seuret. Distributed source seeking via a circular formation of agents under communication constraints. IEEE Transactions on Control of Network Systems, 3(2):104-115, 2015.

[7] Hossein Chehardoli and Ali Ghasemi. Adaptive centralized/decentralized control and identification of 1-d heterogeneous vehicular platoons based on constant time headway policy. IEEE Transactions on Intelligent Transportation Systems, 19(10):3376-3386, 2018.

[8] Hossein Chehardoli and Ali Ghasemi. Formation control of longitudinal vehicular platoons under generic network topology with heterogeneous time delays. Journal of Vibration and Control, 25(3):655-665, 2019.

[9] F. de Oliveira Souza, L. A. B. Torres, L. A. Mozelli, and A. A. Neto. Stability and formation error of homogeneous vehicular platoons with communication time delays. IEEE Transactions on Intelligent Transportation Systems, 21(10):4338-4349, 2020 .

[10] Ioannis M Delimpaltadakis, Charalampos P Bechlioulis, and Kostas J Kyriakopoulos. Decentralized platooning with obstacle avoidance for car-like vehicles with limited sensing. IEEE Robotics and Automation Letters, 3(2):835-840, 2018. 
[11] Eloy Garcia, Yongcan Cao, and David W Casbeer. Periodic event-triggered synchronization of linear multi-agent systems with communication delays. IEEE Transactions on Automatic Control, 62(1):366-371, 2016.

[12] H. Günther, R. Riebl, L. Wolf, and C. Facchi. Collective perception and decentralized congestion control in vehicular ad-hoc networks. In 2016 IEEE Vehicular Networking Conference (VNC), pages 1-8, 2016.

[13] Robert M. Gray. Toeplitz and circulant matrices: A review. Foundations and Trends® in Communications and Information Theory, 2(3):155-239, 2006.

[14] Gagan Rajesh Gupta and Ness Shroff. Delay analysis for multi-hop wireless networks. In IEEE INFOCOM 2009, pages 2356-2364. IEEE, 2009.

[15] Uwe Küchler and Beatrice Mensch. Langevins stochastic differential equation extended by a time-delayed term. Stochastics and Stochastic Reports, 40(1-2):23-42, 1992.

[16] Hanjun Li, Chunhan Feng, Henry Ehrhard, Yijun Shen, Bernardo Cobos, Fangbo Zhang, Karthik Elamvazhuthi, Spring Berman, Matt Haberland, and Andrea L Bertozzi. Decentralized stochastic control of robotic swarm density: Theory, simulation, and experiment. In 2017 IEEE/RSJ International Conference on Intelligent Robots and Systems (IROS), pages 4341-4347. IEEE, 2017.

[17] Shancang Li, Li Da Xu, and Shanshan Zhao. 5g internet of things: A survey. Journal of Industrial Information Integration, $10: 1-9,2018$.

[18] Lili Ma and Naira Hovakimyan. Cooperative target tracking in balanced circular formation: Multiple uavs tracking a ground vehicle. In 2013 American Control Conference, pages 5386-5391. IEEE, 2013.

[19] Brandon J Moore and Carlos Canudas-de Wit. Source seeking via collaborative measurements by a circular formation of agents. In Proceedings of the 2010 American control conference, pages 6417-6422. IEEE, 2010.

[20] Emre Ozfatura, Baturalp Buyukates, Deniz Gunduz, and Sennur Ulukus. Age-based coded computation for bias reduction in distributed learning, 2020.

[21] Hangli Ren, Guangdeng Zong, Linlin Hou, and Yi Yang. Finite-time resilient decentralized control for interconnected impulsive switched systems with neutral delay. ISA transactions, 67:19-29, 2017.

[22] Fabrizio Schiano, Antonio Franchi, Daniel Zelazo, and Paolo Robuffo Giordano. A rigidity-based decentralized bearing formation controller for groups of quadrotor uavs. In 2016 IEEE/RSJ International Conference on Intelligent Robots and Systems (IROS), pages 5099-5106. IEEE, 2016.

[23] Weisong Shi, Jie Cao, Quan Zhang, Youhuizi Li, and Lanyu Xu. Edge computing: Vision and challenges. IEEE internet of things journal, 3(5):637-646, 2016.

[24] Wenqi Shi, Sheng Zhou, Zhisheng Niu, Miao Jiang, and Lu Geng. Joint device scheduling and resource allocation for latency constrained wireless federated learning. IEEE Transactions on Wireless Communications, 20(1):453-467, 2020.

[25] Amr Suleiman, Zhengdong Zhang, Luca Carlone, Sertac Karaman, and Vivienne Sze. Navion: A fully integrated energyefficient visual-inertial odometry accelerator for autonomous navigation of nano drones. In 2018 IEEE Symposium on VLSI Circuits, pages 133-134. IEEE, 2018.

[26] Shanhe Yi, Cheng Li, and Qun Li. A survey of fog computing: concepts, applications and issues. In Proceedings of the 2015 workshop on mobile big data, pages 37-42, 2015.

[27] Quan Yuan, Jingyuan Zhan, and Xiang Li. Outdoor flocking of quadcopter drones with decentralized model predictive control. ISA transactions, 71:84-92, 2017.

[28] Xiaofeng Zong, Tao Li, and Ji-Feng Zhang. Consensus conditions of continuous-time multi-agent systems with time-delays and measurement noises. Automatica, 99:412 - 419, 2019. 